JAMA | Original Investigation

\title{
Effects of Improvisational Music Therapy vs Enhanced Standard Care on Symptom Severity Among Children With Autism Spectrum Disorder The TIME-A Randomized Clinical Trial
}

\begin{abstract}
Łucja Bieleninik, PhD; Monika Geretsegger, PhD; Karin Mössler, Drscmus; Jörg Assmus, PhD; Grace Thompson, PhD; Gustavo Gattino, PhD; Cochavit Elefant, PhD; Tali Gottfried, PhD; Roberta Igliozzi, PhD; Filippo Muratori, MD; Ferdinando Suvini, MA; Jinah Kim, PhD; Mike J. Crawford, MD; Helen Odell-Miller, PhD; Amelia Oldfield, PhD; Órla Casey, MA; Johanna Finnemann, MRes; John Carpente, PhD; A-La Park, MSc; Enzo Grossi, MD; Christian Gold, PhD; for the TIME-A Study Team
\end{abstract}

IMPORTANCE Music therapy may facilitate skills in areas affected by autism spectrum disorder (ASD), such as social interaction and communication.

OBJECTIVE To evaluate effects of improvisational music therapy on generalized social communication skills of children with ASD.

DESIGN, SETTING, AND PARTICIPANTS Assessor-blinded, randomized clinical trial, conducted in 9 countries and enrolling children aged 4 to 7 years with ASD. Children were recruited from November 2011 to November 2015, with follow-up between January 2012 and November 2016.

INTERVENTIONS Enhanced standard care $(n=182)$ vs enhanced standard care plus improvisational music therapy $(n=182)$, allocated in a 1:1 ratio. Enhanced standard care consisted of usual care as locally available plus parent counseling to discuss parents' concerns and provide information about ASD. In improvisational music therapy, trained music therapists sang or played music with each child, attuned and adapted to the child's focus of attention, to help children develop affect sharing and joint attention.

MAIN OUTCOMES AND MEASURES The primary outcome was symptom severity over 5 months, based on the Autism Diagnostic Observation Schedule (ADOS), social affect domain (range, 0-27; higher scores indicate greater severity; minimal clinically important difference, 1). Prespecified secondary outcomes included parent-rated social responsiveness. All outcomes were also assessed at 2 and 12 months.

RESULTS Among 364 participants randomized (mean age, 5.4 years; 83\% boys), 314 (86\%) completed the primary end point and 290 (80\%) completed the last end point. Over 5 months, participants assigned to music therapy received a median of 19 music therapy, 3 parent counseling, and 36 other therapy sessions, compared with 3 parent counseling and 45 other therapy sessions for those assigned to enhanced standard care. From baseline to 5 months, mean ADOS social affect scores estimated by linear mixed-effects models decreased from 14.08 to 13.23 in the music therapy group and from 13.49 to 12.58 in the standard care group (mean difference, 0.06 [ $95 \% \mathrm{Cl},-0.70$ to 0.81$] ; P=.88$ ), with no significant difference in improvement. Of 20 exploratory secondary outcomes, 17 showed no significant difference.

CONCLUSIONS AND RELEVANCE Among children with autism spectrum disorder, improvisational music therapy, compared with enhanced standard care, resulted in no significant difference in symptom severity based on the ADOS social affect domain over 5 months. These findings do not support the use of improvisational music therapy for symptom reduction in children with autism spectrum disorder.

TRIAL REGISTRATION isrctn.org Identifier: ISRCTN78923965

JAMA. 2017;318(6):525-535. doi:10.1001/jama.2017.9478
Editorial page 523

Supplemental content

CME Quiz at

jamanetwork.com/learning and CME Questions page 571
Author Affiliations: Author affiliations are listed at the end of this article.

Group Information: The TIME-A Study Team members are listed at the end of this article.

Corresponding Author: Christian Gold, PhD, GAMUT-The Grieg Academy Music Therapy Research Centre, Uni Research Health, Uni Research, Postbox 7805, 5020 Bergen, Norway (christian.gold@uni.no). 
A utism spectrum disorder (ASD) is characterized by persistent deficits in social communication and interaction and restricted, repetitive behaviors and interests. ${ }^{1}$ ASD affects about $1 \%$ of the general population and is associated with substantial disability and economic loss. ${ }^{2,3}$ A variety of approaches to improve the core deficits and lives of people with ASD have been developed, including behavioral, developmental, educational, and medical interventions, ${ }^{4,5}$ but the strength of evidence for reducing autism severity is low for most interventions. ${ }^{4}$

In the first description of autism, Kanner ${ }^{6}$ noted that many children with autism had a strong preference for music. Music therapy seeks to exploit the potential of music as a medium for social communication. ${ }^{7-9}$ In improvisational music therapy, client and therapist spontaneously create music using singing, playing, and movement; music is understood in the widest sense. It is a developmental, child-centered approach in which a music therapist follows the child's focus of attention, behaviors, and interests to facilitate development in the child's social communicative skills. ${ }^{10}$ In 2017 there were about 7000 music therapists in the United States ${ }^{11}$ and 6000 in Europe. ${ }^{12}$ Randomized trials have suggested positive effects of music therapy on social interaction, joint attention, and parent-child relationships. ${ }^{13}$ Evidence on longer-term effects and dose-effect relations is lacking, and evidence that effects observed within therapy sessions generalize to other settings, situations, and people needs confirmation. ${ }^{13}$ The objective of this study was to evaluate effects of improvisational music therapy on generalized social communication skills of children with ASD.

\section{Methods}

\section{Trial Design}

The Trial of Improvisational Music Therapy's Effectiveness for Children With Autism (TIME-A) was an assessor-blinded, international, multicenter (10 centers), parallel-group, pragmatic randomized clinical trial that compared improvisational music therapy (hereafter referred to as music therapy) added to enhanced standard care (usual care plus parent counseling; hereafter referred to as standard care) with standard care alone for improving social communicative skills in children with ASD. Ethics approval was obtained by the relevant ethics committees in each of the 9 countries (Australia, Austria, Brazil, Israel, Italy, Korea, Norway, United Kingdom, United States). Written informed consent was obtained from parents or guardians. An independent data and safety monitoring committee monitored safety and examined interim efficacy results. The study protocol was published separately ${ }^{14}$ and is also available in Supplement 1.

\section{Participants and Trial Procedures}

Trial procedures were pilot tested..$^{15}$ Children aged 4 years to 6 years, 11 months and meeting criteria for ASD according to the International Statistical Classification of Diseases and Related Health Problems, Tenth Revision ${ }^{16}$ were enrolled between November 2011 and November 2015 and followed up

\section{Key Points}

Question Does improvisational music therapy improve symptom severity of children with autism spectrum disorder?

Findings In a randomized clinical trial of 364 children in 9 countries, mean autism severity, as measured on the Autism Diagnostic Observation Schedule, social affect domain, over 5 months, was reduced from 14.08 to 13.23 in the improvisational music therapy group and from 13.49 to 12.58 in the enhanced standard care group, which included parent counseling and other available interventions, a nonsignificant mean difference of 0.06

Meaning In children with autism spectrum disorder, music therapy did not result in significant improvements in mean symptom scores compared with enhanced standard care.

from January 2012 to November 2016. Exclusion criteria were serious sensory disorders (blindness, deafness) and having received music therapy in the last 12 months. Diagnosis was confirmed using the ASD cutoff of the Autism Diagnostic Observation Schedule $(\mathrm{ADOS})^{17}$ and cutoffs on 2 of the 3 main domains of the Autism Diagnostic Interview-Revised. ${ }^{18}$ Cognitive ability was assessed quantitatively using a standardized IQ test or categorically by clinical judgment if the child was unable to complete a formal test. Participating parents or guardians completed the Social Responsiveness Scale (SRS) ${ }^{19}$ and two 100-mm visual analog scales for quality of life of the participant and the family. Parents also reported demographics and concomitant treatments. Follow-up assessments at 2, 5, and 12 months after randomization included ADOS, SRS, quality of life, and concomitant treatments; at 12 months, we also assessed success of blinding and reason for potential dropout.

After consent and baseline assessments, participants were randomly assigned to 1 of 3 interventions: low-intensity (once per week) or high-intensity (3 times per week) music therapy added to standard care, or standard care alone, over a period of 5 months. Participants were free to attend any type of treatment or therapeutic interventions but were asked not to attend music therapy outside the study context.

Individuals were randomly assigned according to a computer-generated randomization list with a ratio 1:1:2 (lowintensity music therapy:high-intensity music therapy: standard care), stratified by site and with randomly varying block sizes of 4 and 8, which was prepared by an investigator with no clinical involvement. A coordinator (E.B.) with no clinical involvement checked eligibility and baseline data before handing out the randomization via an online system.

All data were stored within an electronic database management system on a secure server with password-controlled access (OpenClinica, version 3.3) and double-entered independently. Changes to the protocol compared with its published version are shown in eTable 1 in Supplement 2.

\section{Interventions}

Improvisational music therapy was offered in outpatient settings (clinics, kindergartens, family homes) in 30-minute oneto-one sessions (possibly joined by family members). Depending on the randomization to high- or low-intensity music 
therapy, either 3 weekly sessions (a higher dose, common in some countries) or 1 weekly session (the de facto standard in many countries) were offered for a period of 5 months, a duration deemed sufficient to notice development. Thirty qualified music therapists (21 women; mean age, 34.7 years [range, 23-55 years]; mean experience as music therapists, 7.3 years [range, 0-30 years]) conducted the therapy, following a set of consensus principles developed for the study. ${ }^{10}$ Therapists developed joint musical activities (singing or instrumental play) individually with each child, based on the child's focus of attention, using improvisation techniques such as synchronizing, mirroring, or grounding. These activities aimed to develop and enhance affect sharing and joint attention, which are associated with development of social competencies in ASD. ${ }^{14}$ Sessions were videotaped or audiotaped for assessment of fidelity. ${ }^{10,14}$ Two independent raters assessed 606 randomly selected 3-minute segments from 63 participants.

Enhanced standard care consisted of the routine care available at the site, plus three 60-minute sessions of parent counseling (at 0, 2, and 5 months). Twenty-four professionals experienced with ASD (20 women; clinical psychologists, social workers, or music therapists) offered to discuss parents' concerns and provide information about ASD, based on principles described in the protocol. ${ }^{14}$ The purpose of the design was to provide a minimal intervention and to increase adherence and equipoise. Sessions were videotaped when possible. Parents reported other service use.

\section{Outcomes}

The primary outcome was the social affect score of the ADOS, ${ }^{17}$ a measure of autism symptom severity, over 5 months. The ADOS is a semistructured, standardized observation instrument. Designed as a diagnostic tool, it has also been used to measure intervention outcomes. ${ }^{20-25}$ One of 3 modules and 1 of 5 scoring algorithms was chosen, depending on language abilities and age. Of a total of 28 to 31 ADOS items, 10 are used to calculate the $\mathrm{ADOS}$ social affect score. Items can range from 0 to 2 or 3; in line with an earlier trial, ${ }^{25}$ the full range of item scores from 0 to 3 was retained to improve sensitivity to change. We applied the same module across time points for each child to ensure consistency. ${ }^{14}$ The ADOS social affect score, constructed as the sum of the relevant items for the social affect domain, can range from 0 to 27 (in module 3; 0-24 in modules 1 and 2), with higher scores indicating greater severity. We assumed a minimal clinically important difference (MCID) of 1 point. ${ }^{14}$ We used the most current version available in all countries and languages. ${ }^{17}$ Formal training in ADOS assessments was available in some but not all countries. All assessors in Australia, Korea, and the United Kingdom had completed research-level qualification (the highest level); those in Israel, Italy, Norway, and the United States had at least clinicallevel qualification (the basic level). In Austria and Brazil, no certification was available, but we used experienced ADOS raters. Of the 23 assessors, 10 had research training and 10 had clinical training. We used assessors from a different location who were not normally involved with the child to ensure blinding. Success of blinding was verified by asking assessors if and how they had discovered the child's allocation.
Prespecified secondary outcomes were the ADOS social affect score over 2 and 12 months and the SRS total scale and its 5 subscales over 2, 5, and 12 months. The SRS is a parentrated measure and was therefore not blinded. A total of 65 items (each ranging from 0 ["not true"] to 3 ["almost always true"]) assess the severity of ASD symptoms occurring in natural social settings, as observed by parents, for a total score of 0 to 195. A score of 85 or above provides strong evidence of the presence of an ASD and is recommended for clinical settings. ${ }^{19}$ The subscales are social awareness ( 8 items), social cognition (12), social communication (22), social motivation (11), and autistic mannerisms (12). ${ }^{19}$ An additional outcome, cost-effectiveness, will be reported separately.

Post hoc outcomes, all at 2, 5, and 12 months, included the ADOS total score and subscales; two 100-mm visual analog scales for parent-reported quality of life of the child and of the family as a whole ( 0 = worst to 100 = best possible quality of life); and parent-reported adverse events including hospitalization.

\section{Statistical Analyses}

The study was originally conceived as a group sequential design with 4 interim analyses ${ }^{14}$ but was stopped after the first prespecified analysis (see Results). Power was calculated for the 2-group comparison to detect an MCID of 1 point for ADOS social affect score. Although there is no consensus MCID for this outcome, a 1-point difference in ADOS social affect score (typical SD, $\approx 5)^{25}$ was chosen because it would correspond to Cohen $d=0.20$ (a small effect size). Previous music therapy trials had found effect sizes of $d=0.50$ for gestural and $d=0.36$ for verbal communicative skills, ${ }^{26}$ whereas a larger trial of another intervention ${ }^{25}$ had found an effect size of $d=0.24$ on the ADOS. ${ }^{14}$ As conducted, the study had $42 \%$ power to detect a difference of 1 point, or $80 \%$ power for a difference of 1.6 points.

The main statistical analysis ${ }^{14}$ followed a modified intention-to-treat (ITT) principle, including all participants who had data for at least 1 follow-up time point in the group to which they had originally been randomized. Analyses compared mean change on the primary outcome in longitudinal models, following confirmation of normality. We calculated linear mixedeffects models with maximum likelihood estimation, both unadjusted and adjusted for site as a random effect, for the main 2-group comparison and the additional 3-group comparison including intensity of music therapy, with treatment effects represented as interaction effects (time $\times$ group). Sensitivity analyses for the primary outcome and comparison tested the influence of missing data (linear models on 50 data sets multiply imputed using diagnosis, age, and site) and therapist effects (linear mixed-effects model with music therapist as a random effect nested within site). Prespecified subgroup analyses were conducted for age and ASD subtype, using linear models with interaction tests.

Secondary outcomes were analyzed using linear mixedeffects models. Because no adjustments for multiple testing were made, all secondary outcomes were regarded as exploratory. No statistical analysis was conducted for exploratory adverse events.

In a post hoc responder analysis, ${ }^{27}$ we compared the proportion of participants who improved by at least the MCID for 
the ADOS social affect score at 5 months. In this binary ITT analysis, we included all participants randomized, assuming no improvements for missing data. We calculated risk ratios with 2 -sided 95\% confidence intervals using Wald unconditional maximum likelihood estimation for the ITT sample and the per-protocol sample. All tests were 2 -sided, with a significance level of $5 \%$. All statistical analyses were conducted using R version 3.3.1 (http://www.r-project.org).

\section{Results}

Of 702 children assessed for eligibility, 315 (109 ineligible; 206 declined) were excluded prior to baseline assessments, and another 23 were found ineligible at baseline, before randomization. A total of 364 participants were randomized to music therapy or standard care (182 participants each) (Figure 1). Of the 182 children enrolled to music therapy, 90 were randomized to high-intensity music therapy and 92 to low-intensity music therapy. The data and safety monitoring committee examined the first interim efficacy analysis in September 2015. Although the formal criterion for early stopping was not met, the study team decided to stop recruitment, in a decision that included considerations of limited funding and therefore limited likelihood of successful and timely additional recruitment.

Baseline characteristics were well balanced between groups (Table 1). Of the 364 participants, 302 were boys, 301 were diagnosed with childhood autism, and 165 had low cognitive levels (IQ < 70). Twelve participants (3\%) had prior experience with music therapy. Fifty participants (14\%) were lost to the 5-month follow-up (Figure 1). Baseline characteristics of those who dropped out at 5 months were similar to those who were followed up (eTable 2 in Supplement 2).

Blinding of assessors was broken unintentionally for 20 participants (15 in the music therapy group and 5 in the standard care group), usually due to a parent or other person inadvertently mentioning the intervention. There was no evidence of broken or subverted allocation concealment (eFigure 1 in Supplement 2).

Concomitant interventions, provided as part of standard care, included speech and language therapy or communication training (58\%), sensory-motor therapy (including occupational therapy and physiotherapy [41\%]), and a number of other therapies, which often continued over the course of the trial (eTable 3 in Supplement 2). The median number of sessions of all concomitant interventions (not including parent counseling or improvisational music therapy) over the 5 -month intervention period was 45 in those allocated to standard care, compared with 36 sessions in those allocated to music therapy (high-intensity music therapy, 31; low-intensity music therapy, 40). The parents of 317 (87\%) of all participants participated in counseling; the median number of sessions was 3 in all groups (Figure 1); fidelity was adequate (eTable $4 \mathrm{~A}$ in Supplement 2).

Of those allocated to music therapy, $171(94 \%)$ received music therapy, with a median of 19 sessions over the 5-month period (high-intensity music therapy, 34; low-intensity music therapy, 15) (Figure 1). Missed sessions were typically attributable to holidays or illness (eFigure 2 in Supplement 2). However, some parents had difficulties bringing their child to therapy 3 times a week. Treatment fidelity according to the improvisational music therapy manual ${ }^{10}$ was overall adequate in the majority of sessions (eTable 4 and eFigure 3 in Supplement 2). Of those allocated to standard care, none received music therapy during the 5-month intervention period. However, $1 \%$ in each group received music therapy outside the study before the 12-month follow-up (Figure 1).

\section{Primary Outcome}

From baseline to 5 months, mean scores of ADOS social affect estimated by linear mixed-effects models decreased from 14.08 to 13.23 in the music therapy group and from 13.49 to 12.58 in the standard care group (mean difference, music therapy vs standard care, 0.06 [95\% CI, -0.70 to 0.81 ]; $P=.88$ ), with no significant difference in improvement (eTable 5 in Supplement 2). The observed means (Table 2) differ slightly from those estimated with linear mixed-effects models, which use data from all time points at once. Differences in the models adjusted for site were also nonsignificant (eTable 5 in Supplement 2). No significant differences were found between high- or lowintensity music therapy and standard care (eTable 6 in Supplement 2). Changes over time are shown in Figure 2. Prespecified subgroup analyses did not suggest different effects of music therapy vs standard care attributable to age $(P=.30)$ or ASD subtype $(P=.87)$.

\section{Secondary Outcomes}

Of 20 prespecified exploratory secondary outcomes, most showed no significant difference (eTables 5 and 6 in Supplement 2). ADOS social affect scores over 2 and 12 months and SRS total scores over 2, 5, and 12 months are also shown in Table 2 and Figure 2. Small but nominally significant effects were found in several SRS subscales (in linear mixedeffects models either adjusted or unadjusted for site)-music therapy was associated with greater improvements than standard care in social motivation over 5 months and autistic mannerisms over 2 and 12 months (eTable 5 in Supplement 2). In the 3-group comparison, low-intensity music therapy, compared with standard care, was associated with greater improvements in social awareness at 2 months; high-intensity music therapy, compared with standard care, was associated with greater improvements in autistic mannerisms over 5 months (eTable 6 in Supplement 2).

\section{Exploratory Analyses and Outcomes}

No significant difference between music therapy and standard care was seen in the sensitivity analysis using multiple imputation (mean difference, music therapy vs standard care, 0.06 [95\% CI, -0.61 to 0.72]; $P=.87$ ) or including the music therapist as a random effect (mean difference, music therapy vs standard care, 0.05 [95\% CI, -0.71 to 0.80 ]; $P=.90$ ).

Post hoc responder analyses indicated a higher proportion of improvement in ADOS social affect at 5 months in the music therapy group (95/182 [52\%]) than in the standard care group (76/182 [42\%]) (risk ratio, 1.25 [95\% CI, 1.00 to 1.56]; 
90 Randomized to receive high-intensity music therapy plus enhanced standard care for $5 \mathrm{mo}^{\mathrm{b}, \mathrm{c}}$

85 Received any music therapy as randomized (median, 34 [IQR, 26-46] sessions; 77 received $\geq 15$ sessions)

5 Did not receive music therapy

83 Received parent counseling (median, 3 [IQR, 2-3] sessions) 7 Did not receive parent counseling

12 Withdrew

5 Unhappy with allocation

1 Child health issue

1 Difficult situation at home

2 Other reason

3 Unknown reason

$$
\checkmark
$$

67 Assessed at $2 \mathrm{mo}$

78 Assessed at $5 \mathrm{mo}$

72 Assessed at $12 \mathrm{mo}$

78 Included in primary analysis

12 Excluded (withdrew)
702 Children assessed for eligibility

315 Excluded

109 Did not meet inclusion criteria

45 Too old $(\geq 7 \mathrm{y})$

4 Too young $(<4 \mathrm{y})$

26 Did not have ASD

30 Received music therapy in

last $12 \mathrm{mo}$

4 Parental language barrier ${ }^{\mathrm{a}}$

138 Logistical reasons

66 Unable to attend required

appointments

58 Located too far away to receive

ongoing services

14 Moved out of area

32 Not interested in participating

30 Could not be contacted or did

not attend

6 Current family or health issues

387 Assessed at baseline

364 Randomized

92 Randomized to receive low-intensity music therapy plus enhanced standard care for $5 \mathrm{mo}^{\mathrm{c}, \mathrm{d}}$

85 Received any music therapy as randomized (median, 15 [IQR, 13-17] sessions; 57 received $\geq 15$ sessions)

7 Did not receive music therapy

83 Received parent counseling (median, 3 [IQR, 2-3] sessions) 9 Did not receive parent counseling

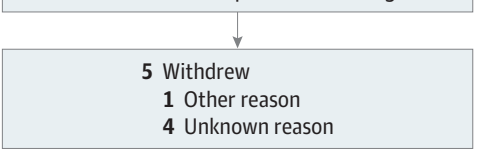

4 Unknown reaso

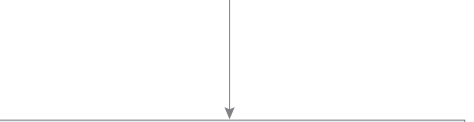

78 Assessed at 2 mo

87 Assessed at $5 \mathrm{mo}$

82 Assessed at $12 \mathrm{mo}$

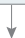

87 Included in primary analysis

5 Excluded (withdrew)
182 Randomized to receive enhanced standard care for $5 \mathrm{moc}^{\mathrm{c}}$

151 Received parent counseling as randomized (median, 3 [IQR, 1-3] sessions)

31 Did not receive parent counseling

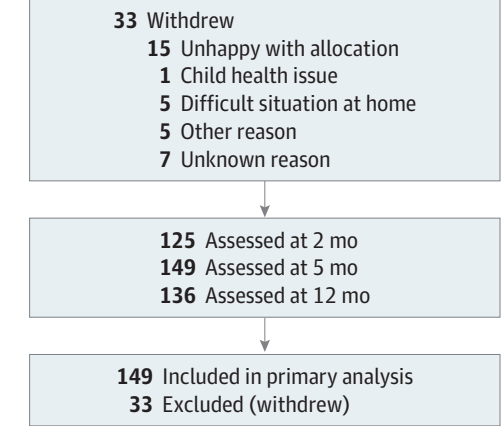

Numbers assessed are based on valid data for the primary outcome. The intermediate assessment at 2 months was optional at UK sites. ASD indicates autism spectrum disorder; IQR, interquartile range.

${ }^{\text {a }}$ Refers to migrant families unable to complete questionnaires or complete clinical interviews because of insufficient command of the country's main language(s).
${ }^{\mathrm{b}}$ High-intensity music therapy comprised 3 music therapy sessions per week (up to 60 sessions).

c Enhanced standard care comprised parent counseling ( 3 sessions) plus usual care. ${ }^{\mathrm{d}}$ Low-intensity music therapy comprised 1 music therapy session per week (up to 20 sessions). risk difference, 0.10 [95\% CI, 0.00 to 0.21$], P=.047$ ). The proportion of improvement was higher in participants who received at least 15 music therapy sessions (78/134 [58\%]) than in those who received standard care (76/182 [42\%]) (risk ratio, 1.39 [95\% CI, 1.11 to 1.74]; risk difference, 0.16 [95\% CI, 0.05 to 0.27 ]; $P=.004)$.
Among the post hoc outcomes, mean changes in participants' quality of life at 5 months were significantly more positive in the high-intensity music therapy group than in the standard care group, but no significant differences were seen in other outcomes or comparisons (eTables 5 and 6 in Supplement 2). 


\begin{tabular}{|c|c|c|c|c|c|c|}
\hline \multirow[b]{3}{*}{ Characteristics } & & & \multicolumn{4}{|c|}{ Improvisational Music Therapy } \\
\hline & \multicolumn{2}{|c|}{ Enhanced Standard Care } & \multicolumn{2}{|c|}{ High Intensity } & \multicolumn{2}{|c|}{ Low Intensity } \\
\hline & No. & Value & No. & Value & No. & Value \\
\hline Age, mean (SD), y & 182 & $5.4(0.9)$ & 90 & $5.4(0.9)$ & 92 & $5.5(0.8)$ \\
\hline Boys, No. (\%) & 182 & 149 (81.9) & 90 & $78(86.7)$ & 92 & $71(77.2)$ \\
\hline $\begin{array}{l}\text { Native speaker of the country's } \\
\text { main language, No. (\%) }\end{array}$ & 182 & $152(83.5)$ & 90 & $78(86.7)$ & 92 & $74(80.4)$ \\
\hline $\begin{array}{l}\text { Maternal education, } \\
\text { No. (\%) }\end{array}$ & 180 & & 89 & & 89 & \\
\hline$<12$ y (less than high school) & & $17(9.4)$ & & $9(10.1)$ & & $10(11.2)$ \\
\hline $\begin{array}{l}\geq 12 \text { y (equivalent to } \\
\text { high school) }\end{array}$ & & $64(35.6)$ & & $37(41.6)$ & & $31(34.8)$ \\
\hline University & & $84(46.7)$ & & $38(42.7)$ & & $42(47.2)$ \\
\hline Unknown & & $15(8.3)$ & & $5(5.6)$ & & $6(6.7)$ \\
\hline Paternal education, №. (\%) & 178 & & 87 & & 89 & \\
\hline$<12$ y (less than high school) & & $27(15.2)$ & & $11(12.6)$ & & $12(13.5)$ \\
\hline $\begin{array}{l}\geq 12 \text { y (equivalent to } \\
\text { high school) }\end{array}$ & & $54(30.3)$ & & $33(37.9)$ & & $28(31.5)$ \\
\hline University & & $77(43.3)$ & & $34(39.1)$ & & $40(44.9)$ \\
\hline Unknown & & $20(11.2)$ & & $9(10.3)$ & & $9(10.1)$ \\
\hline $\begin{array}{l}\text { Maternal employment, } \\
\text { No. (\%) }\end{array}$ & 180 & & 89 & & 89 & \\
\hline $\begin{array}{l}\text { Unemployed } \\
\text { or social support }\end{array}$ & & $29(16.1)$ & & $10(11.2)$ & & $9(10.1)$ \\
\hline Working part time & & $41(22.8)$ & & $29(32.6)$ & & $22(24.7)$ \\
\hline Working full time & & $36(20.0)$ & & $13(14.6)$ & & $18(20.2)$ \\
\hline Homemaker & & $61(33.9)$ & & 30 (33.7) & & $32(36)$ \\
\hline Other & & $2(1.1)$ & & $3(3.4)$ & & $2(2.2)$ \\
\hline Unknown & & $11(6.1)$ & & $4(4.5)$ & & $6(6.7)$ \\
\hline $\begin{array}{l}\text { Paternal employment, } \\
\text { No. (\%) }\end{array}$ & 179 & & 88 & & 89 & \\
\hline $\begin{array}{l}\text { Unemployed } \\
\text { or social support }\end{array}$ & & $7(3.9)$ & & $9(10.2)$ & & $3(3.4)$ \\
\hline Working part time & & $11(6.1)$ & & $5(5.7)$ & & $2(2.2)$ \\
\hline Working full time & & $136(76.0)$ & & $58(65.9)$ & & $70(78.7)$ \\
\hline Homemaker & & $2(1.1)$ & & 0 & & $3(3.4)$ \\
\hline Other & & $5(2.8)$ & & $4(4.5)$ & & $2(2.2)$ \\
\hline Unknown & & $18(10.1)$ & & $12(13.6)$ & & $9(10.1)$ \\
\hline Adults in household, No. (\%) & 174 & & 87 & & 84 & \\
\hline 1 & & $21(12.1)$ & & $17(19.5)$ & & $9(10.7)$ \\
\hline 2 & & $137(78.7)$ & & $62(71.3)$ & & $72(85.7)$ \\
\hline$>2$ & & $16(9.2)$ & & $8(9.2)$ & & $3(3.6)$ \\
\hline Siblings in family, No. (\%) & 172 & & 87 & & 83 & \\
\hline None & & $56(32.6)$ & & $16(18.4)$ & & $18(21.7)$ \\
\hline 1 & & $77(44.8)$ & & $43(49.4)$ & & $43(51.8)$ \\
\hline$>1$ & & $39(22.7)$ & & $28(32.2)$ & & $22(26.5)$ \\
\hline Diagnosis, №. (\%) & 182 & & 90 & & 92 & \\
\hline $\begin{array}{l}\text { Childhood autism } \\
\text { (ICD-10 code F84.0) }\end{array}$ & & $151(83.0)$ & & $78(86.7)$ & & $72(78.3)$ \\
\hline $\begin{array}{l}\text { Atypical autism } \\
\text { (ICD-10 code F84.1) }\end{array}$ & & $3(1.6)$ & & 0 & & 0 \\
\hline $\begin{array}{l}\text { Asperger syndrome } \\
\text { (ICD-10 code F84.5) }\end{array}$ & & $8(4.4)$ & & $2(2.2)$ & & $4(4.3)$ \\
\hline PDD (ICD-10 code F84.9) & & $20(11.0)$ & & $10(11.1)$ & & $16(17.4)$ \\
\hline $\begin{array}{l}\text { Previous music therapy, } \\
\text { No. (\%) }\end{array}$ & 177 & $8(4.5)$ & 89 & $7(7.9)$ & 90 & $1(1.1)$ \\
\hline
\end{tabular}




\begin{tabular}{|c|c|c|c|c|c|c|}
\hline \multirow[b]{3}{*}{ Characteristics } & \multirow{2}{*}{\multicolumn{2}{|c|}{ Enhanced Standard Care }} & \multicolumn{4}{|c|}{ Improvisational Music Therapy } \\
\hline & & & & High Intensity & \multicolumn{2}{|c|}{ Low Intensity } \\
\hline & No. & Value & No. & Value & No. & Value \\
\hline \multicolumn{7}{|l|}{$\mathrm{ADOS}^{\mathrm{a}}$} \\
\hline Module, No. (\%) & 182 & & 90 & & 92 & \\
\hline 1 & & $103(56.6)$ & & $65(72.2)$ & & $56(60.9)$ \\
\hline 2 & & $73(40.1)$ & & $25(27.8)$ & & $31(33.7)$ \\
\hline 3 & & $6(3.3)$ & & 0 & & $5(5.4)$ \\
\hline \multicolumn{7}{|l|}{ Score, mean (SD) } \\
\hline Total & 181 & $17.4(5.2)$ & 90 & $18.3(5.1)$ & 92 & $17.7(5.7)$ \\
\hline Social affect & 182 & $13.5(4.3)$ & 90 & $14.4(4.5)$ & 92 & $13.8(4.5)$ \\
\hline Language and communication & 182 & $3.3(1.4)$ & 90 & $3.4(1.5)$ & 92 & $3.3(1.6)$ \\
\hline Reciprocal social interaction & 182 & $10.2(3.5)$ & 90 & $11.0(3.5)$ & 92 & $10.4(3.7)$ \\
\hline Restricted and repetitive behavior & 181 & $3.9(2.0)$ & 90 & $3.9(2.0)$ & 92 & $3.9(2.2)$ \\
\hline \multicolumn{7}{|l|}{ SRS score, mean (SD) ${ }^{\mathrm{b}}$} \\
\hline Total & 179 & $96.1(29.5)$ & 89 & $95.5(26.1)$ & 91 & $96.5(28.5)$ \\
\hline Social awareness & 181 & $12.4(4.1)$ & 90 & $12.2(3.6)$ & 92 & $12(4.1)$ \\
\hline Social cognition & 182 & $18.7(5.9)$ & 90 & $18(5.3)$ & 92 & $18.4(6.5)$ \\
\hline Social communication & 182 & $32.1(10.1)$ & 90 & $31.9(9.8)$ & 92 & $32.4(10.6)$ \\
\hline Social motivation & 182 & $15(6.2)$ & 90 & $15(5.0)$ & 92 & $14.6(5.7)$ \\
\hline Autistic mannerisms & 182 & $17.6(7.1)$ & 90 & $18.1(7.3)$ & 92 & $18.7(6.9)$ \\
\hline \multicolumn{7}{|l|}{ Quality of life, mean (SD) ${ }^{\mathrm{c}}$} \\
\hline Participant & 181 & $71.1(18.7)$ & 88 & $72.3(18.5)$ & 88 & $71.9(18.5)$ \\
\hline Family & 181 & $68(19.4)$ & 87 & $68.7(21.2)$ & 89 & $67.8(19.6)$ \\
\hline $\begin{array}{l}\text { Parent working reduced hours due } \\
\text { to the child's needs (in percent } \\
\text { of full-time work), mean (SD) }\end{array}$ & 101 & $69.1(31.1)$ & 42 & $67.9(29.6)$ & 38 & $69.5(30.5)$ \\
\hline IQ source, No. (\%) ${ }^{d}$ & 182 & & 90 & & 92 & \\
\hline KABC & & $3(1.6)$ & & $3(3.3)$ & & $2(2.2)$ \\
\hline Other standardized test & & $107(58.8)$ & & $50(55.6)$ & & $53(57.6)$ \\
\hline Clinical judgment & & $72(39.6)$ & & $37(41.1)$ & & $37(40.2)$ \\
\hline IQ, standardized test, mean (SD) ${ }^{\mathrm{e}}$ & 108 & $76.1(27.4)$ & 50 & $73.4(27.5)$ & 53 & $75.9(22.7)$ \\
\hline Mental retardation (IQ <70), No. (\%) & 180 & $84(46.7)$ & 87 & $44(50.6)$ & 89 & $40(44.9)$ \\
\hline \multicolumn{7}{|l|}{ ADI-R score, mean $(S D)^{f}$} \\
\hline Reciprocal social interaction & 182 & $18.2(5.8)$ & 90 & $18.8(5.6)$ & 92 & $18.0(5.9)$ \\
\hline Language and communication & 182 & $13.1(4.3)$ & 90 & $13.3(4.2)$ & 92 & $12.5(4.0)$ \\
\hline $\begin{array}{l}\text { Repetitive behaviors } \\
\text { and interests }\end{array}$ & 182 & $5.9(2.5)$ & 90 & $5.9(2.4)$ & 92 & $5.8(2.1)$ \\
\hline Early onset & 182 & $3.9(1.1)$ & 90 & $4.1(1.0)$ & 92 & $4(1.1)$ \\
\hline Child care, No. (\%) & 182 & & 90 & & 92 & \\
\hline Attends school & & $112(61.5)$ & & $56(62.2)$ & & $61(66.3)$ \\
\hline Full-time care $(\geq 7 \mathrm{~h} / \mathrm{d})$ & & $40(22.0)$ & & $19(21.1)$ & & $21(22.8)$ \\
\hline Part-time care $(<7 \mathrm{~h} / \mathrm{d})$ & & $19(10.4)$ & & $13(14.4)$ & & $6(6.5)$ \\
\hline None of the above & & $11(6.0)$ & & $2(2.2)$ & & $4(4.3)$ \\
\hline \multirow{2}{*}{\multicolumn{3}{|c|}{$\begin{array}{l}\text { Abbreviations: ADI-R, Autism Diagnostic Interview-Revised; ADOS, Autism } \\
\text { Diagnostic Observation Schedule; ICD-10, International Statistical Classification } \\
\text { of Diseases and Related Health Problems, Tenth Revision; KABC, Kaufman } \\
\text { Assessment Battery for Children; SRS, Social Responsiveness Scale; PDD, } \\
\text { pervasive developmental disorder unspecified. } \\
\text { a Higher scores indicate greater severity (ranges of possible scores: total, 0-37; } \\
\text { social affect, 0-27; language and communication, 0-9; reciprocal social } \\
\text { interaction, 0-19; restricted and repetitive behavior, 0-10). }\end{array}$}} & \multicolumn{4}{|c|}{$\begin{array}{l}{ }^{\mathrm{c}} \text { Assessed using visual analog scale. Higher scores indicate better quality of life } \\
\text { (range of possible scores, 0-100; O indicates worst possible and } 100 \text { best } \\
\text { possible quality of life). }\end{array}$} \\
\hline & & & \multicolumn{4}{|c|}{$\begin{array}{l}\text { e Higher scores indicate greater cognitive ability. Scores around } 100 \text { indicate } \\
\text { normal intelligence; scores below } 70 \text { indicate mental retardation. }\end{array}$} \\
\hline \multicolumn{3}{|c|}{$\begin{array}{l}\text { b Higher scores indicate greater severity (ranges of possible scores: total, 0-195; } \\
\text { social awareness, 0-24; social cognition, 0-36; social communication, 0-66; } \\
\text { social motivation, 0-33; autistic mannerisms, 0-36). }\end{array}$} & \multicolumn{4}{|c|}{$\begin{array}{l}{ }^{f} \text { Higher scores indicate greater severity (ranges of possible scores: reciprocal } \\
\text { social interaction, 0-30; language and communication, 0-26; repetitive } \\
\text { behaviors and interests, 0-12; early onset, 0-5). }\end{array}$} \\
\hline
\end{tabular}




\begin{tabular}{|c|c|c|c|c|c|c|c|c|}
\hline & \multicolumn{4}{|c|}{ Observed Values } & \multicolumn{4}{|c|}{ Change From Baseline } \\
\hline & \multicolumn{2}{|c|}{ Enhanced Standard Care } & \multicolumn{2}{|c|}{ Improvisational Music Therapy } & \multicolumn{2}{|c|}{ Enhanced Standard Care } & \multicolumn{2}{|c|}{ Improvisational Music Therapy } \\
\hline & No. & Mean $(95 \% \mathrm{Cl})$ & No. & Mean $(95 \% \mathrm{Cl})$ & No. & Mean $(95 \% \mathrm{Cl})$ & No. & Mean $(95 \% \mathrm{Cl})$ \\
\hline \multicolumn{9}{|c|}{ ADOS Social Affect ${ }^{\mathrm{b}}$} \\
\hline Baseline & 182 & $\begin{array}{l}13.49 \\
(12.86 \text { to } 14.12)\end{array}$ & 182 & $\begin{array}{l}14.08 \\
\text { (13.43 to } 14.73)\end{array}$ & & & & \\
\hline $2 \mathrm{mo}$ & 125 & $\begin{array}{l}12.58 \\
\text { (11.81 to } 13.34)\end{array}$ & 145 & $\begin{array}{l}13.88 \\
\text { (13.10 to } 14.65)\end{array}$ & 125 & $\begin{array}{l}-0.44 \\
(-0.99 \text { to } 0.11)\end{array}$ & 145 & $\begin{array}{l}-0.21 \\
(-0.69 \text { to } 0.26)\end{array}$ \\
\hline $5 \mathrm{mo}$ & 149 & $\begin{array}{l}12.45 \\
\text { (11.71 to } 13.19)\end{array}$ & 165 & $\begin{array}{l}13.27 \\
\text { (12.55 to } 14.00)\end{array}$ & 149 & $\begin{array}{l}-0.83 \\
(-1.38 \text { to }-0.28)\end{array}$ & 165 & $\begin{array}{l}-0.87 \\
(-1.38 \text { to }-0.35)\end{array}$ \\
\hline $12 \mathrm{mo}$ & 136 & $\begin{array}{l}11.72 \\
\text { (10.95 to } 12.49)\end{array}$ & 154 & $\begin{array}{l}12.60 \\
(11.81 \text { to } 13.40)\end{array}$ & 136 & $\begin{array}{l}-1.60 \\
(-2.27 \text { to }-0.93)\end{array}$ & 154 & $\begin{array}{l}-1.51 \\
(-2.05 \text { to }-0.96)\end{array}$ \\
\hline \multicolumn{9}{|c|}{ SRS Total ${ }^{\mathrm{c}}$} \\
\hline Baseline & 179 & $\begin{array}{l}96.08 \\
\text { (91.76 to } 100.41 \text { ) }\end{array}$ & 180 & $\begin{array}{l}96.03 \\
\text { (92.04 to } 100.01 \text { ) }\end{array}$ & & & & \\
\hline $2 \mathrm{mo}$ & 120 & $\begin{array}{l}93.73 \\
\text { (88.22 to } 99.25)\end{array}$ & 137 & $\begin{array}{l}91.98 \\
\text { (87.53 to } 96.42)\end{array}$ & 119 & $\begin{array}{l}0.33 \\
(-3.04 \text { to } 3.70)\end{array}$ & 135 & $\begin{array}{l}-2.52 \\
(-5.25 \text { to } 0.21)\end{array}$ \\
\hline $5 \mathrm{mo}$ & 129 & $\begin{array}{l}93.29 \\
\text { (88.01 to } 98.56)\end{array}$ & 142 & $\begin{array}{l}89.15 \\
\text { (84.61 to } 93.70)\end{array}$ & 128 & $\begin{array}{l}-1.97 \\
(-5.60 \text { to } 1.66)\end{array}$ & 141 & $\begin{array}{l}-5.23 \\
(-8.44 \text { to }-2.03)\end{array}$ \\
\hline $12 \mathrm{mo}$ & 126 & $\begin{array}{l}88.64 \\
(83.38 \text { to } 93.91)\end{array}$ & 132 & $\begin{array}{l}86.46 \\
(81.20 \text { to } 91.72)\end{array}$ & 124 & $\begin{array}{l}-5.06 \\
(-8.94 \text { to }-1.19)\end{array}$ & 131 & $\begin{array}{l}-7.37 \\
(-10.95 \text { to }-3.78)\end{array}$ \\
\hline \multicolumn{9}{|c|}{$\begin{array}{l}\text { Abbreviations: ADOS Autism Diagnostic Observation Schedule; SRS, Social } \\
\text { Responsiveness Scale. }\end{array}$} \\
\hline \multicolumn{9}{|c|}{$\begin{array}{l}\text { a Table reports observed values. Estimations from linear-effects models used for } \\
\text { estimating effects are reported in eTables } 5 \text { and } 6 \text { in Supplement } 2 \text {. The } \\
\text { primary outcome (social affect values determined by linear mixed-effects }\end{array}$} \\
\hline
\end{tabular}

\section{Adverse Events}

Hospitalization or other institutional stay was rare at baseline ( 9 assigned to music therapy and 3 assigned to standard care had an institutional stay during the last 2 months). During participation in the study, these rates remained stable in the standard care group ( 3 at 2 months, 3 at 5 months, and 4 at 12 months) and decreased in the music therapy group ( 6 at 2 months, 6 at 5 months, and 4 at 12 months). These institutional stays were typically planned and short-term. No other adverse events or serious adverse events were reported.

\section{Discussion}

In this international, multicenter clinical trial of children with ASD, improvisational music therapy added to enhanced standard care, compared with enhanced standard care alone, resulted in no significant difference in symptom severity based on the ADOS social affect domain over 5 months. Additionally, the amount of improvement in both groups was small and less than the MCID, suggesting that use of improvisational music therapy for children with ASD may not lead to meaningful improvement in symptom severity and may not be warranted for improving autistic symptoms.

Most of the exploratory secondary outcomes were also nonsignificant. The few significant outcomes were small and unlikely to be clinically important. Observed differences in social responsiveness subscales may be artifacts attributable to multiplicity and lack of blinding. The post hoc finding of a higher proportion of responders in the music therapy group compared with the standard care group should be interpreted with caution and may be biased by differential attrition. No dose effect was found in the prespecified analyses.
The findings contrast with those of previous studies. A systematic review of 10 clinical trials concluded that music therapy may help children with ASD to improve skills in areas constituting the core of the condition. ${ }^{13}$ One important difference is that previous trials were limited to 1 local context and 1 or a few therapists, where consistent implementation of interventions may be easier than in a global trial.

Alternatively, methodological differences, such as the choice of a proximal vs a distal outcome, also may explain the difference. No trial of music therapy and very few trials of other psychosocial interventions showed effects on generalized behaviors ${ }^{4}$ using blinded assessments on the ADOS. Assessorblinded trials of parent-mediated interventions have shown mixed results. ${ }^{20,23-25}$ A nonblinded study found effects of a teacher-directed intervention. ${ }^{22}$ Another nonblinded study of a developmental intervention, with more than 1000 treatment hours per child, did not. ${ }^{21}$

Although a large number of treatment hours is considered important in autism interventions, early claims suggesting benefits with early intensive behavioral intervention were not replicated in rigorous randomized evaluations. ${ }^{21,28}$ The burden associated with attending therapy sessions also needs to be considered. In this study, participants assigned to music therapy tended to receive fewer other therapies, and not all were able to attend as frequently as planned, particularly when additional travel was needed.

Although the present trial did not capture the child's experience with improvisational music therapy, it seemed very well accepted by parents, children, and staff. In a qualitative study connected to this trial, parents reported their children's enjoyment and benefit from improvisational music therapy and experienced their own involvement as positive. ${ }^{29}$ This study represented a first attempt to implement music 
A ADOS social affect score

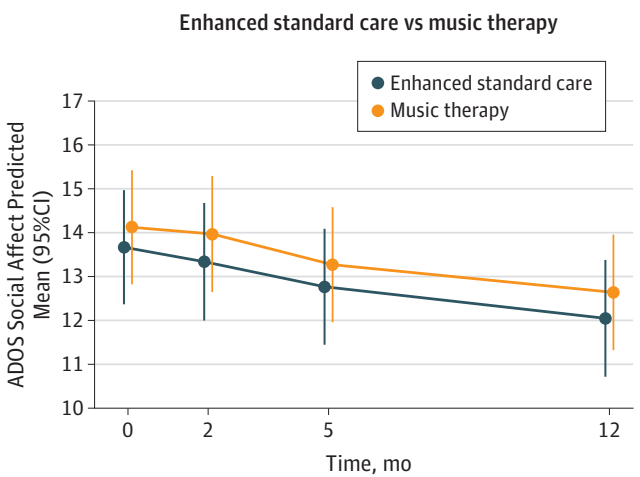

No. of participants

Enhanced standard care $182 \quad 125 \quad 149$

$\begin{array}{llll}\text { Music therapy } & 182 & 145 & 165\end{array}$

B Social Responsiveness Scale

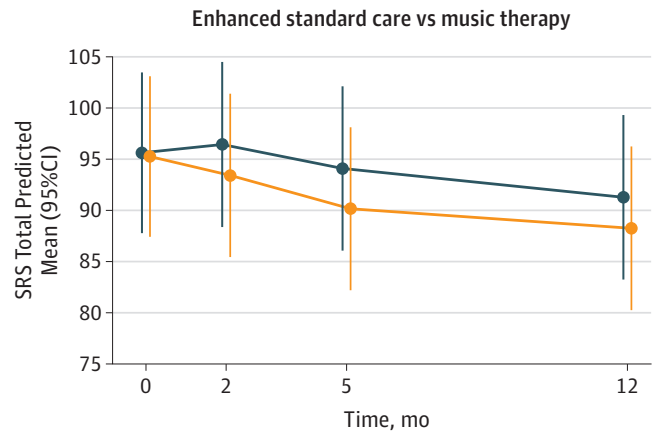

No. of participants

Enhanced standard care $179 \quad 120 \quad 129$

Music therapy

$180 \quad 137$

142

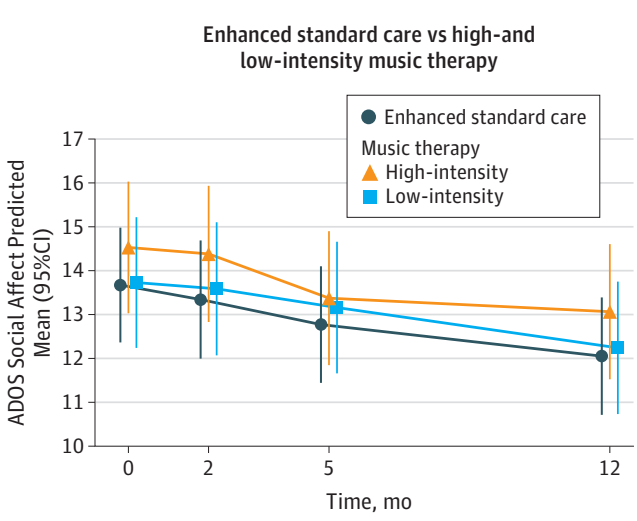

No. of participants

$\begin{array}{lllll}\text { Enhanced standard care } & 182 & 125 & 149 & 136\end{array}$

Music therapy

High-intensity

Low-intensity

$92 \quad 78$

87

78

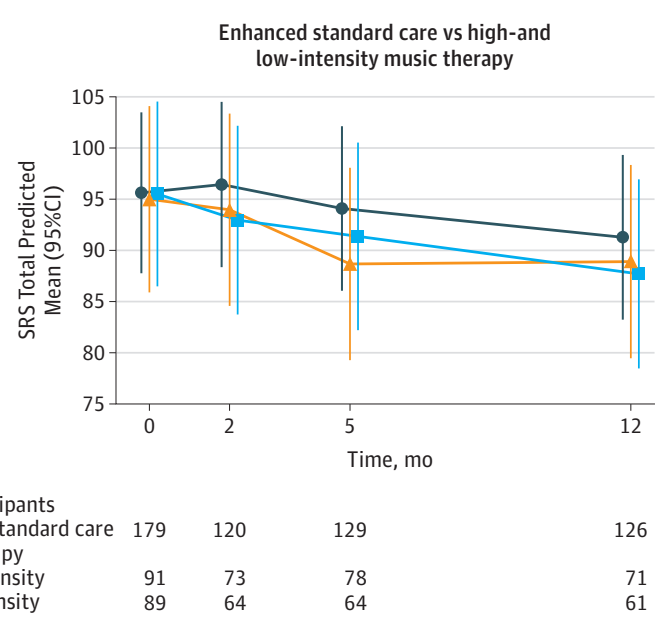

total, range of possible scores, 0 to 195; higher scores indicate greater severity. High-intensity music therapy comprised 3 music therapy sessions per week (up to 60 sessions); low-intensity music therapy comprised 1 music therapy session per week (up to 20 sessions).
No. of participants

$\begin{array}{llll}\text { Enhanced standard care } & 179 & 120 & 129\end{array}$

Music therapy

High-intensity

Low-intensity
Graphs illustrate the predicted values from linear mixed-effects models presented in eTables 5 and 6 in Supplement 2. Autism Diagnostic Observation Schedule (ADOS) social affect score (range of possible scores, 0-27; higher scores indicate greater severity; minimal clinically important difference, 1) over 5 months was the primary outcome. For the Social Responsiveness Scale (SRS) 


\section{Conclusions}

Among children with autism spectrum disorder, improvisational music therapy, compared with enhanced standard care, resulted in no significant difference in symptom severity based on the ADOS social affect domain over 5 months. These findings do not support the use of improvisational music therapy for symptom reduction in children with autism spectrum disorder.

\section{ARTICLE INFORMATION}

Accepted for Publication: July 5, 2017.

Author Affiliations: GAMUT-The Grieg Academy Music Therapy Research Centre, Uni Research Health, Uni Research, Bergen, Norway (Bieleninik, Geretsegger, Mössler, Assmus, Gold); Melbourne Conservatorium of Music, University of Melbourne, Melbourne, Australia (Thompson); Department of Communication and Psychology, Aalborg University, Aalborg, Denmark (Gattino): Postgraduate Program in Child and Adolescent Health, Faculty of Medicine, Rio Grande do Sul Federal University, Porto Alegre, Brazil (Gattino); School for Creative Arts Therapies, University of Haifa, Haifa, Israel (Elefant); Music Therapy Program, David-Yelin College, Jerusalem, Israel (Gottfried); IRCCS Stella Maris Foundation, Pisa, Italy (Igliozzi, Muratori, Suvini); Department of Clinical and Experimental Medicine, University of Pisa, Pisa, Italy (Muratori); Jeonju University, Jeonju, South Korea (Kim); Centre for Psychiatry, Imperial College London, London, United Kingdom (Crawford); Anglia Ruskin University, Cambridge, United Kingdom (Odell-Miller, Oldfield, Casey, Finnemann); Department of Psychiatry, University of Cambridge, Cambridge, United Kingdom (Finnemann); Rebecca Center for Music Therapy at Molloy College, Rockville Centre, New York (Carpente); Personal Social Services Research Unit, LSE Health and Social Care, The London School of Economics and Political Science, London, United Kingdom (Park); Villa Santa Maria Institute, Tavernerio, Italy (Grossi).

Author Contributions: Drs Assmus and Gold had full access to all of the data in the study and take responsibility for the integrity of the data and the accuracy of the data analysis.

Concept and design: Geretsegger, Assmus, Gold.

Acquisition, analysis, or interpretation of data:

Bieleninik, Geretsegger, Mössler, Assmus, Thompson,

Gattino, Elefant, Gottfried, Igliozzi, Muratori, Suvini,

Kim, Crawford, Odell-Miller, Oldfield, Casey,

Finnemann, Carpente, Park, Grossi, Gold.

Drafting of the manuscript: Bieleninik, Geretsegger, Assmus, Gold.

Critical revision of the manuscript for important intellectual content: Geretsegger, Mössler, Assmus, Thompson, Gattino, Elefant, Gottfried, Igliozzi, Muratori, Suvini, Kim, Crawford, Odell-Miller, Oldfield, Casey, Finnemann, Carpente, Park, Grossi, Gold. Statistical analysis: Assmus, Finnemann, Park, Grossi, Gold.

Obtained funding: Geretsegger, Mössler, Thompson, Kim, Crawford, Odell-Miller, Gold. Administrative, technical, or material support: Bieleninik, Geretsegger, Mössler, Thompson, Gattino, Kim, Crawford.

Supervision: Thompson, Gattino, Igliozzi, Muratori, Suvini, Kim, Crawford, Odell-Miller, Oldfield, Carpente, Gold.

Conflict of Interest Disclosures: All authors have completed and submitted the ICMJE Form for Disclosure of Potential Conflicts of Interest and none were reported.
Funding/Support: This trial was funded by the Research Council of Norway (grant 213844, the Clinical Research and Mental Health Programmes) Additional funding was provided by the POLYFON Knowledge Cluster for Music Therapy, The Grieg Academy Department of Music, University of Bergen. Funding for the individual countries came from the following sources: Australia: Melbourne Conservatorium of Music, The University of Melbourne; Austria: Danish Council for Independent Research/Humanities (FKK), Aalborg University, and Faculty of Psychology, University of Vienna; Brazil: Research Incentive Fund (FIPE) of the Hospital de Clínicas de Porto Alegre (HCPA); Italy: IRCCS Stella Maris Foundation, Pisa, and University of Pisa; Korea: Research Institute of Health and Science, Jeonju University; Norway: Health Authority of Western Norway (Helse Vest grant 911800), Bergen municipality (Bergen Culture School), Fjell municipality (Fjell Culture School); United Kingdom: National Institute for Health Research (Health Technology Assessment Programme, National Institute for Health Research grant 12/167/95), Cambridge and Peterborough Foundation National Health Service Trust, The Evelyn Trust, Cambridgeshire Music, Anglia Ruskin University; United States: Molloy College Faculty research scholarship and a collaborative research grant from the Mid-Atlantic Region of the American Music Therapy Association.

Role of the Funder/Sponsor: The funders of the study had no role in design and conduct of the study; collection, management, analysis, and interpretation of the data; and preparation, review, or approval of the manuscript or the decision to submit for publication.

Group Information: TIME-A Study Team: Australia: Music therapists: Anna-Lee Robertson, MMusThrp, Jo Rimmer, BMusThrp, Grace Thompson, PhD; Clinical assessor: Melina Roberts, MMus; Parent counselor: Grace Thompson, PhD (University of Melbourne). Austria: Music therapists: Agnes Burghardt-Distl, MMag, Monika Geretsegger, MMMag, PhD (private practice); Clinical assessors: Sonja Metzler, Mag, Carolin Steidl, Mag (Österreichische Autistenhilfe); Parent counselor: Monika Geretsegger, MMMag, PhD (private practice). Brazil: Music therapist and parent counselor: Gustavo Andrade de Araujo, PhD (Universidade Federal do Rio Grande do Sul); Clinical assessors: Renata Rocha Kieling, PhD (Santa Casa Hospital), Gabriela Damasceno Ferreira (clinical psychologist), Barbara Backes, PhD, Regina Zanon, PhD (Universidade Federal do Rio Grande do Sul); ADI-R supervisor: Cleonice Bosa, PhD (Universidade Federal do Rio Grande do Sul). Rudimar Riesgo, PhD (Universidade Federal do Rio Grande do Sul) helped with translating and validating the SRS and with obtaining permission for using the ADOS, ADI-R, and SRS in Brazil. Israel: Music therapists and parent counselors: Tali Gottfried, PhD (private practice), Rivka Grossbard, MA (University of Haifa); Clinical assessors: Efrat Eshchar, MA, Edwa Friedlander, MA (University of Haifa). Italy: Music therapists: Stefano Cainelli, diploma in music therapy (University of Trento), Giulio Collavoli, MA, Marco Giusti, MS (IRCCS Stella Maris Foundation, Pisa); Clinical assessors: Fabio Apicella, PhD, Romina Cagiano, MS, Roberta Igliozzi, PhD, Patrizia Masoni, MD, Antonio Narzisi, PhD, Gloria Scarselli, MD, Raffaella Tancredi, MD (IRCCS Stella Maris Foundation, Pisa), Arianna Bentenuto, PhD, Alessandro Carolli, MS, Annarita Contaldo, MD, Michela Zaninelli, MS (University of Trento); Parent counselors: Sara Baldini, PhD, Ferdinando Suvini, MA (IRCCS Stella Maris Foundation, Pisa), Paola Venuti, PhD (University of Trento). Korea: Music therapists: Silvina Choi, MA, Sungha Hwang, MA, Su-A Kim, MA, Ji- Yeon Lee, MA (Love'n Family Music Therapy Center); Clinical assessor: HyunKyung Lee, MA, Eun-Chung Lim, MA (RudolfThe Korea Institute for Children's Social Development); Clinical assessor, advisor, help with recruitment: Yun-Joo Koh, PhD (Rudolf-The Korea Institute for Children's Social Development); Parent counselors: In-Yong Lee, PhD (Gachon University), In-Ryung Song, PhD, Ji-Yeon Lee, MA (Love'n Family Music Therapy Center). Norway: Music therapists and parent counselors: Trond Dahle, MA (PPS Sør, Bergen), Åsta Rosendahl Knudsen, MA (Bergen Culture School), Olena Svatyeva, MA (Fjell Culture School); Parent counselor: Karin Mössler, Drscmus (Uni Research); Clinical assessors: Elin Antoniussen, Stine Mjåtvedt, Line Beate Ulstein, Kjellaug Grøsvik (all candidates in psychology; Haukeland University Hospital); Advisors: Irene Elgen, MD (Haukeland University Hospital), Gun Iversen (candidate in psychology; Haukeland University Hospital). United Kingdom: Music therapists: Laura Blauth, MA, Alexandra Georgaki, MA, Órla Casey, MA (Anglia Ruskin University), Belinda Lydon, MA, Pavlina Papadopoulou, MA, Grace Watts, MA (Central and North West London NHS Foundation Trust); Music therapy supervisors: Claire Grant, MA, Anna Maratos, MS (Central and North West London NHS Foundation Trust), Stephen Sandford, MA (East London NHS Foundation Trust), Amelia Oldfield, PhD (Anglia Ruskin University); Clinical assessors: Sarah Faber, MA, Johanna Finnemann, MA, MRes (Anglia Ruskin University), Lavanya Thana, BS (Imperial College London); Parent counselors: Adewale Adeoye, MBBS (Cambridge and Peterborough NHS Foundation Trust), Laura Blauth, MA, Órla Casey, MA, Alexandra Georgaki, MA, Sonia Yin, MA (Anglia Ruskin University), Arshad Faridi, MBBS, Belinda Lydon, MA, Rania Moussa, MBBS (Central and North West London NHS Foundation Trust); Study administrator: Amy Claringbold, BSc (Imperial College London). United States: Music therapists: Tal Gur, MA (Brooklyn Conservatory of Music), Jesse Asch, MS, Michael Kelliher, MS, Jill Mulholland, MS, Gabriela Ortiz, MS, Suzanne Sorel, DA (Molloy College); Clinical assessors: Kim Gilbert, PhD (Hofstra University), Laura De Gennaro, PsyD, Alan Wenderoff, PsyD (Molloy College); Parent counselors: Audra Cerruto, PhD, Talita Ferrara, MS, Laura Kestemberg, PsyD, Ellana Sanders, MA (Molloy College); Filmers/data archivists: Diana Abourafeh, BS, Jon Fessenden, MS (Molloy College), and Molloy College music therapy 
students and graduate assistants; Adviser: Stella Manne, PhD (Molloy College); Administrative assistant: Stephanie Cestaro (Molloy College).

\section{Reproducible Research Statement: Deidentified} individual participant data for this study are available in the Norwegian Centre for Research Data (NSD) public repository at http://dx.doi.org/10 .18712/NSD-NSD2466-V1.

Disclaimer: The views and opinions expressed therein are those of the authors and do not necessarily reflect those of the Health Technology Assessment programme, National Institute for Health Research, National Health Service, or the Department of Health.

Additional Contributions: We thank all families participating in the study and the referring professionals. Maj-Britt Posserud, MD (Haukeland University Hospital), and Karin Schumacher, Drscmus (Berlin University of the Arts), helped with the design of the study. Jan-Erik Askildsen, Dr oecon (Uni Research and University of Bergen), Barbara Barrett, PhD (King's College London), Egil Kjerstad, PhD (Uni Research), and Ingrid Zechmeister-Koss, Drrer.soc.oec, MA (European Centre for Social Welfare Policy and Research), helped with specific aspects concerning concomitant treatments and economic analyses. Synne Rotvold Røli and Kjetil Andreas Nydal (University of Bergen) helped with assessment of treatment fidelity. Marko Nouwens, MA (Uni Research), and Jo Dugstad Wake, PhD (Uni Research), helped with data management. Leif Edvard Aarø, PhD (Norwegian Institute of Public Health, Department of Health Promotion), Tine Nordgreen, PhD (Division of Psychiatry, Haukeland University Hospital; Department of Clinical Psychology, Faculty of Psychology, University of Bergen), and Otto R. Smith, PhD (Department of Health Promotion, Norwegian Institute of Public Health), served on the data monitoring committee. Laura Fusar-Poli, MD (University of Pavia), provided valuable comments on an earlier version of the manuscript. With the exception of Ms Røli,

Mr Nydal, and Mr Nouwens, none of these people received compensation for their role in the study. Drs Assmus and Gold conducted and are responsible for the data analysis; Dr Grossi provided additional analyses.

\section{REFERENCES}

1. American Psychiatric Association. Diagnostic and Statistical Manual of Mental Disorders. 5th ed. Arlington, Va: American Psychiatric Publishing; 2013.

2. Baxter AJ, Brugha TS, Erskine HE, Scheurer RW, Vos T, Scott JG. The epidemiology and global burden of autism spectrum disorders. Psychol Med. 2015;45(3):601-613.

3. Buescher AV, Cidav Z, Knapp M, Mandell DS. Costs of autism spectrum disorders in the United Kingdom and the United States. JAMA Pediatr. 2014;168(8):721-728.

4. Weitlauf AS, McPheeters ML, Peters B, et al. Therapies for Children With Autism Spectrum
Disorder: Behavioral Interventions Update: Comparative Effectiveness Review No. 137. Rockville, MD: Agency for Healthcare Research and Quality; 2014.

5. Wong $C$, Odom $\mathrm{SL}$, Hume KA, et al. Evidence-based practices for children, youth, and young adults with autism spectrum disorder: a comprehensive review. J Autism Dev Disord. 2015; 45(7):1951-1966.

6. Kanner L. Autistic disturbances of affective contact. Nerv Child. 1943;2:217-250.

7. Alvin J. Music Therapy for the Autistic Child. Oxford, United Kingdom: Oxford University Press; 1978.

8. Stern DN. Forms of Vitality: Exploring Dynamic Experience in Psychology and the Arts. Oxford, United Kingdom: Oxford University Press; 2010.

9. Malloch S, Trevarthen C. Communicative Musicality. Oxford, United Kingdom: Oxford University Press; 2008.

10. Geretsegger M, Holck U, Carpente JA, Elefant C, Kim J, Gold C. Common characteristics of improvisational approaches in music therapy for children with autism spectrum disorder: developing treatment guidelines. J Music Ther. 2015;52(2): 258-281.

11. Certification Board for Music Therapists (CBMT) website. http://www.cbmt.org. Accessed May 30 , 2017.

12. European Music Therapy Confederation (EMTC) website. http://www.emtc-eu.com/about-emtc. Accessed May 30, 2017.

13. Geretsegger M, Elefant C, Mössler KA, Gold C. Music therapy for people with autism spectrum disorder. Cochrane Database Syst Rev. 2014;6(6): CD004381.

14. Geretsegger M, Holck U, Gold C. Randomised controlled trial of improvisational music therapy's effectiveness for children with autism spectrum disorders (TIME-A): study protocol. BMC Pediatr. 2012;12(2):2.

15. Geretsegger M, Holck U, Bieleninik $Ł$, Gold C. Feasibility of a trial on improvisational music therapy for children with autism spectrum disorder. J Music Ther. 2016;53(2):93-120.

16. World Health Organization. International Statistical Classification of Diseases and Related Health Problems. 10th ed. Geneva, Switzerland: World Health Organization; 1992.

17. Gotham K, Risi S, Pickles A, Lord C. The Autism Diagnostic Observation Schedule: revised algorithms for improved diagnostic validity. J Autism Dev Disord. 2007;37(4):613-627.

\section{Lord C, Rutter M, Le Couteur A. Autism}

Diagnostic Interview-Revised: a revised version of a diagnostic interview for caregivers of individuals with possible pervasive developmental disorders. J Autism Dev Disord. 1994;24(5):659-685.

19. Constantino JN, Gruber CP. Social Responsiveness Scale (SRS). Los Angeles, CA: Western Psychological Services; 2005.
20. Aldred C, Green J, Adams C. A new social communication intervention for children with autism: pilot randomised controlled treatment study suggesting effectiveness. J Child Psychol Psychiatry. 2004:45(8):1420-1430.

21. Dawson G, Rogers S, Munson J, et al. Randomized, controlled trial of an intervention for toddlers with autism: the Early Start Denver Model. Pediatrics. 2010;125(1):e17-e23.

22. Howlin P, Gordon RK, Pasco G, Wade A, Charman T. The effectiveness of Picture Exchange Communication System (PECS) training for teachers of children with autism: a pragmatic, group randomised controlled trial. J Child Psychol Psychiatry. 2007;48(5):473-481.

23. Pickles A, Le Couteur $A$, Leadbitter $K$, et al. Parent-mediated social communication therapy for young children with autism (PACT): long-term follow-up of a randomised controlled trial. Lancet. 2016;388(10059):2501-2509.

24. Solomon R, Van Egeren LA, Mahoney G, Quon Huber MS, Zimmerman P. PLAY Project Home Consultation intervention program for young children with autism spectrum disorders: a randomized controlled trial. J Dev Behav Pediatr. 2014;35(8):475-485.

25. Green J, Charman T, McConachie H, et al; PACT Consortium. Parent-mediated communicationfocused treatment in children with autism (PACT): a randomised controlled trial. Lancet. 2010;375 (9732):2152-2160.

26. Gold C, Wigram T, Elefant C. Music therapy for autistic spectrum disorder. Cochrane Database Syst Rev. 2006;(2):CD004381.

27. McGlothlin AE, Lewis RJ. Minimal clinically important difference: defining what really matters to patients. JAMA. 2014;312(13):1342-1343.

28. Reichow B. Overview of meta-analyses on early intensive behavioral intervention for young children with autism spectrum disorders. J Autism Dev Disord. 2012;42(4):512-520.

29. Blauth LK. Improving mental health in families with autistic children: benefits of using video feedback in parent counselling sessions offered alongside music therapy. Health Psychol Rep. 2017;5 (2):138-150.

30. Mössler K, Schmid W. What's this adorable noise? relational qualities in music therapy with children with autism. Nord J Music Ther. 2016;25 (suppl):51-51.

31. Gottfried T, Thompson G, Carpente J, Gattino G. Music in everyday life by parents with their children with autism. Nord J Music Ther. 2016;25(suppl):8990.

32. Silberman S. NeuroTribes: The Legacy of Autism and the Future of Neurodiversity. New York, NY: Penguin Random House LLC; 2015. 